\title{
Effect of attentional selection on working memory for depth in a retro-cueing paradigm
}

\author{
Zhuolun $\mathrm{Li}^{1} \cdot$ Mengxuan Tong ${ }^{1} \cdot$ Shiting Chen ${ }^{1} \cdot$ Jiehui Qian $^{1}$ (i)
}

Accepted: 1 December 2020 / Published online: 7 January 2021

(C) The Psychonomic Society, Inc. 2021

\begin{abstract}
Recent studies have shown that the temporary storage and manipulation of depth information (working memory for depth; WMd) is largely different from that of visual information in a 2D context (visual working memory; VWM). Although there has been abundant evidence on VWM showing that cueing a memory item during retention could bias attention to its internal representation and thus improves its memory performance (a retro-cue effect), it is unknown whether such an effect differs for WMd that is nested in a 3D context compared with that in a conventional 2D context. Here, we used a change detection task to investigate the effect of attentional selection on WMd by testing several types of retro-cue. The memory array consisted of items positioned at various stereoscopic depth planes, and a cue was presented during retention. Participants needed to make judgments on whether the depth position of target (one memory item) had changed. Our study showed reliable valid retro-cue benefits but no invalid retro-cue cost, indicating that the relational information may be registered in WMd to prevent a strategical removal of the unattended item. There was also a slight improvement in memory performance for cueing depth order compared with that for cueing other feature dimensions or 2D locations. The attentional effect on memory representation in a 3D context is different from that in a $2 \mathrm{D}$ context, and the divergence may suggest the distinctive nature of working memory for depth.
\end{abstract}

Keywords Working memory $\cdot$ Attention $\cdot$ Retro-cue effect $\cdot$ Stereoscopic depth

Working memory is considered as a cognitive workspace that allows us to temporarily hold and manipulate sensory information. For decades, verbal working memory and visual working memory (VWM), which are two subsystems of the working memory model responsible for retention and manipulation of verbal and visual information, have been studied extensively (for review, see Baddeley, 2003; Luck \& Vogel, 2013). Recently, several studies investigated the role of depth information played in VWM (Chunharas, Rademaker, Sprague, \& Brady, 2019; Qian, Li, Wang, Liu, \& Lei, 2017; Qian, Zhang, Wang, Li, \& Lei, 2018; Reeves \& Lei, 2014; Xu $\&$ Nakayama, 2007). In addition, researchers began to explore how depth information is stored in working memory (Qian \& Zhang, 2019; Qian, Li, Zhang, \& Lei, 2020; Reeves \& Lei, 2017; Zhang, Gao \& Qian, 2020), and their findings have raised increasing attention.

Jiehui Qian

qianjh3@mail.sysu.edu.cn

1 Department of Psychology, Sun Yat-Sen University, Guangzhou 510006, China
These studies have shown that our ability of retaining depth information is severely limited and is distinct from retaining visual information. For example, compared with nearly perfect memory performance for up to four items (Luck \& Vogel, 1997) or five two-dimensional (2D) spatial locations (Simons, 1996), the accuracy for holding one depth position in a change detection task was below 80\% (Qian \& Zhang, 2019), and the retention of numerals that associated with depths for a set size of one was poor (Reeves \& Lei, 2017). In addition to its capacity limitation, the stored depth representations are found to be inherently relational (Qian et al., 2020), and retention of depth representations seems to be different from VWM. Reeves and Lei (2017) found that memory performance for depth decreases with retention duration for the first $500 \mathrm{~ms}$ and then slightly recovered, demonstrating a U-shaped function of time. There is also a conservative response bias that observers tended to make 'no change' responses when detecting changes in depth (Qian \& Zhang, 2019), compared with a relatively neutral response that was found for detecting changes in color and shape (Qian, Zhang, Lei, Han, \& Li, 2019). Based on the extensive differences, researchers suggest that the nature of working memory for depth information is distinct from what has been reported for working memory for 
visual information and 2D spatial locations in past literature, and further termed it as working memory for depth (WMd; Reeves \& Lei, 2017; also see Qian \& Zhang, 2019; Qian et al., 2020).

Despite that the working memory is putatively known for its limited capacity (e.g., Luck \& Vogel, 1997), in reality, we hardly experience any deficiency in temporarily holding and processing visual information or three-dimensional (3D) spatial information. Although no prior study investigated the attentional effect on WMd, there is abundant evidence showing that several cognitive factors contribute in overcoming the capacity limitation of VWM. For example, attention may 'highlight' a memory representation that is more relevant to the current task, thereby prioritizing its visual processing and maintenance. Research suggests that attention could be directed toward certain locations or representations in VWM, improving memory performance for the relevant items in the focus of attention (Astle, Summerfield, Griffin, \& Nobre, 2012; Griffin \& Nobre, 2003; Murray, Nobre, Clark, Cravo, $\&$ Stokes, 2013; Qian et al., 2019). These studies often used a retro-cue (cueing one memory item during retention) to bias attention to certain internal representations held in VWM, and the resulted change in memory performance (e.g., proportion of correct responses, or an estimate of capacity, $\mathrm{K}$ ) is termed as retro-cue effect. In particular, valid retro-cue benefits refer to the improvement in performance for the cued item compared to the condition of none being cued (neutral cue), while invalid retro-cue costs refer to the decrease in performance for the noncued items compared to the neutral-cue condition (Souza \& Oberauer, 2016).

Researchers suggested that both the retro-cue benefits and costs could be reliably observed under high retro-cue reliability conditions ( $>80 \%$ valid). However, for cues with low reliability or even uninformative (e.g., 50\% valid), retro-cue benefits are still present (though sometimes reduced), but costs may disappear (Berryhill, Richmond, Shay, \& Olson, 2012; Gunseli, Van Moorselaar, Meeter, \& Olivers, 2015; Shimi, Nobre, Astle, \& Scerif, 2014; Souza, Rerko, \& Oberauer, 2015; but also see Wang, Yan, Wang, Olivers, \& Theeuwes, 2017). It seems that retro-cue benefits and costs depend on the reliability of the retro-cue, indicating a strategical deployment of the memory resource based on the predictiveness of the cue. In a recent review, Souza and Oberauer (2016) suggested that a retro-cue might affect the memory performance by strengthening the attended representations in VWM and removing the unattended representations from VWM during the maintenance stage, or by facilitating the decision-making process at the retrieval stage. The 'removal' account is supported by neural evidence (Kuo, Stokes, \& Nobre, 2012), but researchers also suggest that the noncued representations are not irreversibly removed, yet stored in a less 'active' statethe neural activity related to the noncued representations drops to baseline levels and can be reactivated to an 'online' state by a second cue (LaRocque, Lewis-Peacock, Drysdale, Oberauer, \& Postle, 2013; Lewis-Peacock, Drysdale, Oberauer, \& Postle, 2011; van Moorselaar, Olivers, Theeuwes, Lamme, \& Sligte, 2015). This might be because the noncued representations are less accessible when outside the focus of attention and can be recovered once in the focus of attention (Souza \& Oberauer, 2016; Wang, Theeuwes, \& Olivers, 2018). Under high cue reliability conditions, the noncued items are rarely tested and therefore may be worth removing or 'frozen' in a less active state to reduce memory load; but under low cue reliability conditions, removal may not be a good strategy, and therefore little cost is observed (Gunseli et al., 2015).

Since both the cues and the tested memory representations in past research were constrained in a $2 \mathrm{D}$ context and no previous study investigated the retro-cue effect on WMd, it is unclear whether and how attention induced by a retro-cue could aid working memory to deal with its limited ability to process and retain vast 3D spatial information that is simultaneously presented. The retro-cue effect on WMd may differ in terms of the magnitude of valid retro-cue benefits and invalid retro-cue costs, due to the suggested differences in nature between VWM and WMd. Previous study has shown that the relational information, which is indicated by depth order, significantly modulated the memory performance for detecting changes in stereoscopic depth (Qian et al., 2020). Depth order indicates the sequential position in depth of an item, which determines the inherent relationship among depth positions of items. When there is no change in relative depth order, the memory performance for detecting large magnitudes of change in depth locations between the target and probe can still be low; however, when depth order is changed, the performance for detecting small magnitudes of change in depth can be high. The findings suggest that depth position cannot be registered independently, and it is possible that both metric and ordinal depth representations are stored in working memory. Based on these findings, we predict that reliable retro-cue benefits but little or no cost can be observed for WMd, even for cues with high reliability. This is because for invalid trials, it is unlikely to 'remove' a noncued representation to free up memory capacity as in VWM, since the removing process would change the relationship between items and result in disturbance in the ordinal depth representations. In other words, retaining ordinal depth in WMd protects the items from complete removal, or 'frozen' in a less active state.

In this study, we carried out three experiments to test and compare the attentional effects of various types of retro-cues on WMd, and the cue reliability was also manipulated to further confirm the retro-cue effects. We examined three types of cues: feature-based, spatial, and symbolic retro-cues. The former two types of cues are often tested in VWM, with featurebased cues to indicate an item's feature (e.g., color or shape) and spatial cues to indicate a 2D fronto-parallel location to 
guide attention (e.g., using an arrow to point to the location; Griffin \& Nobre, 2003; Heuer \& Schubö, 2016). Although there are debates over the questions of whether the attentional mechanism of feature-based retro-cues is primarily spacebased (Lamy \& Tsal, 2001; McMains, Fehd, Emmanouil, \& Kastner, 2007; Theeuwes, 1989) or related to spatial attention (Poch, Carretie, \& Campo, 2017), recent behavioral and neural evidence reveals that these two modes of selection may rely on different attentional mechanisms (e.g., Heuer \& Schubö, 2016) and that different cortical regions are responsible for the feature-based and spatial selection of working memory representations (Heuer, Schubö, \& Crawford, 2016). Therefore, we tested the feature-based and spatial cues separately to examine their retro-cue effects.

Although less tested, symbolic cues, which usually involve mapping a number onto a specific spatial location, have also been used to examine the retro-cue effects on VWM (Berryhill et al., 2012; Heuer \& Schubö, 2016). Its retro-cue effects are reported to be small (e.g., Heuer \& Schubö, 2016), and sometimes even absent (e.g., Berryhill et al., 2012), possibly due to the greater amount of time or effort it requires to decode symbolic cues than spatial or feature-based cues (Heuer \& Schubö, 2016). In our study, we tested two types of symbolic cue: one that maps a number onto a 2D location as in VWM studies (e.g., Heuer \& Schubö, 2016) and one that maps a number onto the depth order of an item. Unlike 2D symbolic cues, the 3D symbolic cues indicated the spatial relation in depth between a memory item and the observer, and can be crucially relevant to storage of depth representation. Therefore, it is possible that utilizing the relational depth information (i.e., the 3D symbolic cues) could induce stronger retro-cue effects or facilitate memory performance overall. Our study may help us to locate the type of information optimized for accessing or selecting the memory representation in a $3 \mathrm{D}$ context, and the results may shed light on the underlying mechanisms for storage of depth representation.

\section{Experiment 1}

In Experiment 1, we investigated the effects of spatial attention on WMd with a high reliability of retro-cue. We used a change detection paradigm where a set of memory items were briefly presented at various stereoscopic depth positions, and a probe was shown after a retention interval. During the retention, spatial attention was directed either to the 2D location of a memory item using an arrow retro-cue (Experiment 1a) or a 2D symbolic retro-cue (Experiment 1b), or to the depth profile of a memory item using a 3D symbolic retro-cue (Experiments 1a \& 1b).

\section{Method}

\section{Participants}

Twenty-four students from Sun Yat-Sen University (SYSU), who had normal or corrected-to-normal vision, took part in Experiment $1 \mathrm{a}$, and another 24 students participated in Experiment 1b. Twenty-two of them were naïve to the purpose of the study and received payment for their participation; the other two were experienced psychophysical observer. The sample size was based on two criteria: (1) previous literature and (2) a power analysis. Previous study investigating the retro-cue effect on VWM has used a range of sample size from 15 to 24 to look at the retro-cue effects, with a typical effect size of partial $\eta^{2}=0.35$ (Heuer \& Schubö, 2016). In addition, we conducted a power analysis using G*Power (Faul, Erdfelder, Lang, \& Buchner, 2007), which showed that at least nine participants would be required to achieve a statistical power of $90 \%$, at a significance level of 0.05 , to detect a similar effect in our study. Written informed consent was obtained from each participant following a protocol approved by the Institutional Review Board (IRB) of Sun Yat-Sen University (SYSU, Guangzhou, China).

\section{Apparatus and stimuli}

The stimuli were presented against a uniform gray background $\left(102 \mathrm{~cd} / \mathrm{m}^{2}\right)$ through a Wheatstone stereoscope on a pair of 21-inch ViewSonics monitors. The display resolution was set to $1,920 \times 1,080$ pixels, with a refresh rate of $60 \mathrm{~Hz}$. Participants were seated in a dark room with a viewing distance of $75 \mathrm{~cm}$ to complete all the experiments.

The memory array was composed of four blue squares. The squares were arranged on a circular configuration with a radius of $3.5^{\circ}$ from the center of the screen (see Fig. 1). Any two nearest neighboring items were separated by $3.5^{\circ}$. The memory items were presented at various depth planes perpendicular to the line of sight, with one item per depth plane. The depth profile of a memory item was randomly selected from a set of six depth planes without replacement. The depth planes were separated by relative disparities ranging from $-0.50^{\circ}$ to $0.50^{\circ}$ with a step of $0.20^{\circ}$, which corresponded to $-6.87,-4.28,-1.48,1.83,4.80,8.41 \mathrm{~cm}$ from the monitor screen with a typical interpupillary distance of $6.5 \mathrm{~cm}$. These disparities were selected so that the left-eye and right-eye images could be reliably fused, and the items clearly appeared to be separated in depth (Blakemore, 1970). Each item subtended $0.65^{\circ} \times 0.65^{\circ}$ of visual angle. In addition, the items at the farther planes might appear to be larger than those at the nearer ones due to the mechanism of size-distance scaling. We decreased the size of the items by $1 \%$ for each receding plane so that the size of the items at different planes appeared to be the same (for details, see Qian \& Zhang, 2019). 


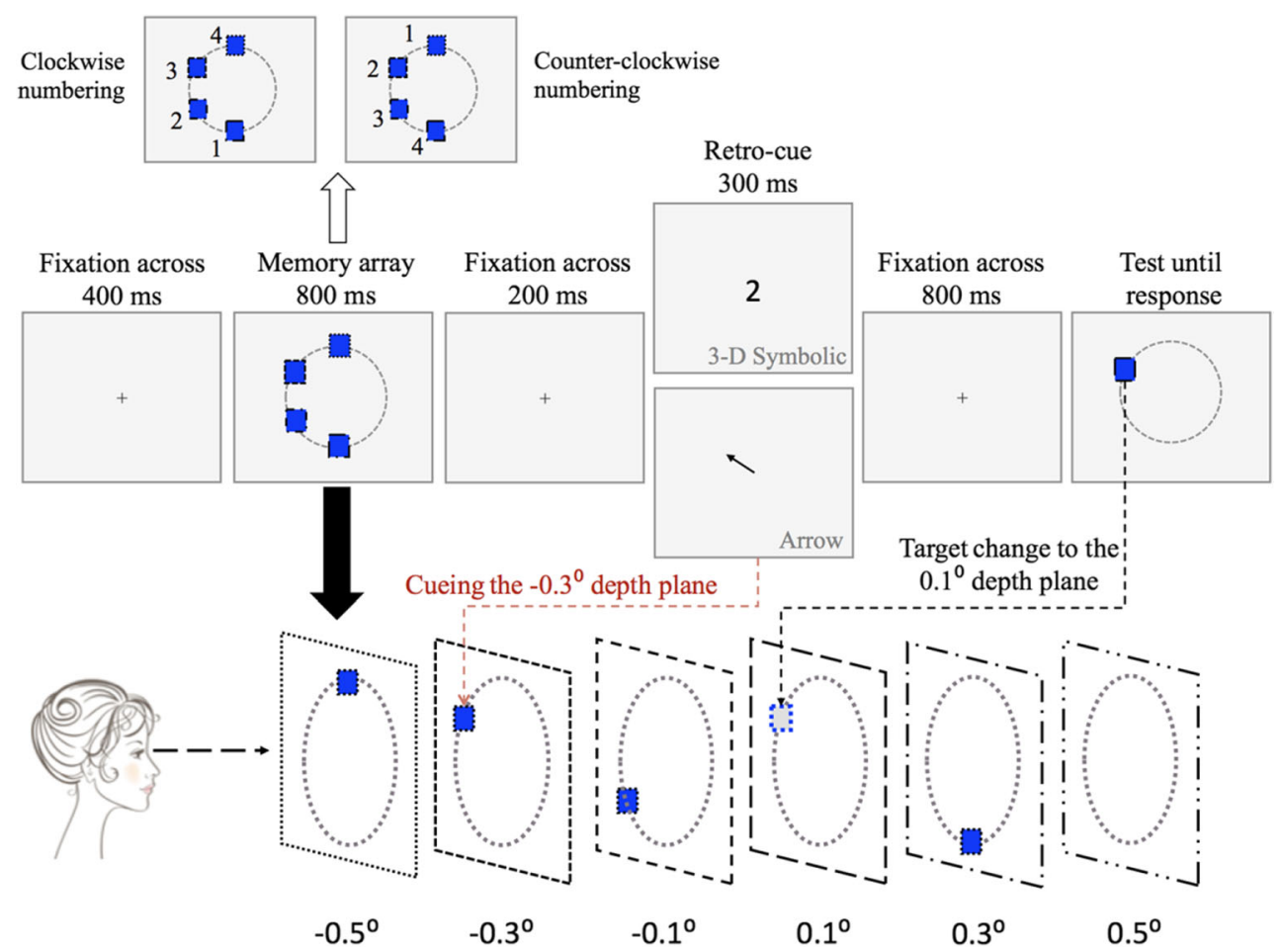

Fig. 1 Stimuli and procedure in Experiment 1a. Top: task sequence. Bottom: the front view and the side view of the memory display. The memory items were arranged in a circular configuration (indicated by a dashed circle) and were outlined with various types of lines to indicate

All retro-cues were centrally placed at the zero-disparity plane. In Experiment 1a, an arrow cue and a 3D symbolic cue were tested and compared; in Experiment 1b, a 2D symbolic cue and the 3D symbolic cue were tested and compared. The arrow cue subtended $0.15^{\circ} \times 0.65^{\circ}$, pointing to one of the $2 \mathrm{D}$ memory locations. The $3 \mathrm{D}$ symbolic cue was a number that subtended $0.30^{\circ} \times 0.65^{\circ}$ mapping onto the depth order of one memory item, with ' 1 ' being the nearest and ' 4 ' being the farthest. The 2D symbolic cue was a number mapping onto the 2D location of one memory item, and the locations were numbered from 1 to 4 clockwise or counterclockwise (see Fig. 1). Half of the participants were tested with the locations numbered clockwise, and the other half counterclockwise. There were also trials with an uninformative neutral cue (a symbol of " $\times$ " subtended $0.30^{\circ} \times 0.30^{\circ}$ ) in each retro-cue type conditions.

\section{Procedure}

Before the experiments, the participants were required to pass a screening task to ensure that they could well perceive the stereoscopic depth (see Qian \& Zhang, 2019, for details). After that, they were trained for a short time (2-5 min) and completed 48 practice trials to get acquainted with the stimuli and the task. In the formal experiment, each block started with a fusion display with a red cross subtended $0.35^{\circ} \times 0.35^{\circ}$ at the their different depth positions. No line or circle was presented in the actual experiment. This figure shows an example of a "change" trial in the valid retro-cue condition

center of the screen. The participants were instructed to fixate at the cross and fuse the left-eye and right-eye images of the red cross until no double image was perceived. They then confirmed the success of fusion by pressing ' 3 ' on the keyboard, and the cross turned black. Trials began after the black cross was presented for $400 \mathrm{~ms}$, and the task sequence is illustrated in Fig. 1. A memory array was presented for 800 $\mathrm{ms}$. Then, after an $800 \mathrm{~ms}$ retention interval, either a valid, an invalid, or a neutral retro-cue was presented for $300 \mathrm{~ms}$, followed by another retention interval of $800 \mathrm{~ms}$. A valid retro-cue indicated a memory item that would be subsequently probed, while an invalid retro-cue indicated a memory item that would not be tested. A neutral cue provided no information on which item would be probed. In the test phase, a probe was shown until response at one of the 2D memory locations (test location). Participants were informed of the cue reliabilities prior to the start of the experiment. They were asked to memorize the depth position of each memory item and to judge whether the item at the test location had changed its depth (press key ' 1 ' for no change and key '2' for change).

In each experiment, each observer completed a total of 480 trials, with 240 trials for each retro-cue type. There were 24 invalid retro-cue trials, 192 valid retro-cue trials (retro-cue reliability $89 \%, 192 / 216$ ), and 24 neutral cue trials. There were $50 \%$ 'change' trials in each experimental condition, on which the target would change to a new depth plane different 
from the ones in the memory array. The two retro-cue types (arrow cue and 3D symbolic cue in Experiment 1a; 2D and 3D symbolic cues in Experiment 1b) were tested in separate experimental blocks, with randomized order across participants.

For data analysis, participants who had a mean accuracy of below $50 \%$ in any of the experimental conditions were considered as careless and were excluded for analysis. In Experiment $1 \mathrm{a}$, four participants were excluded; in Experiment 1b, two were excluded.

For each experiment, we performed a $2 \times 3$ (retro-cue type $\times$ retro-cue validity) repeated-measures ANOVA on accuracyto compare the retro-cue benefits and costs between the two cue types (detection sensitivity, $d^{\prime}$, based on hit and false- alarm rates was also calculated, and the results were generally consistent with those of accuracy and are reported in the Supplemental Materials). The $p$ values of post hoc tests were Bonferroni corrected. We also performed analysis on Bayes factors (BFs) to confirm the statistical results (see Supplemental Materials for detailed reports).

\section{Results and discussion}

\section{Experiment 1a}

The results are shown in Fig. 2, left. There was a significant main effect of retro-cue validity, $F(2,38)=13.75, p<.001$, $\eta_{\mathrm{p}}^{2}=.42$. Post hoc comparisons showed that the performance was significantly better for the valid retro-cues $(M=.77)$ than for the neutral cues $(M=.72), t(19)=2.93, p=.026$, and the invalid retro-cues $(M=.67), t(19)=5.42, p<.001$. No significant difference was found between the neutral and invalid

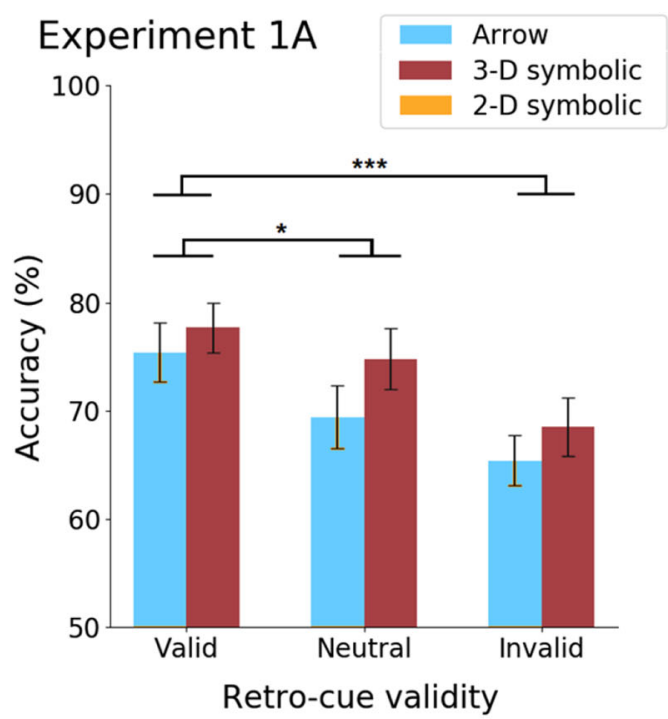

Fig. 2 Results of accuracy in Experiment 1. Left: for the arrow and 3D symbolic retro-cues in Experiment 1a, there were significant retro-cue benefits, but no retro-cue cost. Right: for 2D symbolic and 3D symbolic retro-cues in Experiment $1 \mathrm{~b}$, memory performance was significantly retro-cue conditions, $t(19)=2.39, p=.083$. There was a significant main effect of retro-cue type, $F(1,19)=6.05, p=$ $.024, \eta_{\mathrm{p}}^{2}=.24$. The results showed that the accuracy was significantly better for the $3 \mathrm{D}$ symbolic cues $(M=.73)$ than for the arrow cues $(M=.70)$. No significant interaction between cue type and cue validity was found, $F(2,38)=.41, p=$ $.668, \eta_{\mathrm{p}}^{2}=.02$.

Consistent with our prediction, the results showed significant retro-cue benefits, but no significant retro-cue cost. The accuracy was higher for 3D symbolic cues than for arrow cues, indicating that cueing the same information dimension (depth) as the memory task may produce a benefit. One might suspect that cueing depth order could allow participants to correctly perform the task in a valid trial even without retaining the exact metric depth information. However, we think that it is unlikely for the following reasons: (1) the improvement in performance was also shown for neutral and invalid trials; (2) since only the probe was presented during the test phase and no other item could be served as references, knowing the depth order alone hardly provides any helpful information that could enhance the change detection performance even for valid trials. For participants to correctly respond in a valid trial based solely on depth order, one needs to first accurately construct an internal representation of the whole depth volume tested in our experiment through extensive repeated trials, then locate the possible depth range of the target based on its depth order, and finally it has to be a 'change' trial and the change has to be large enough for a correct guess (for a 'no change' trial the correct guess rate would be at chance). Although we cannot completely rule out this possibility, there was a slim chance for a trial to satisfy

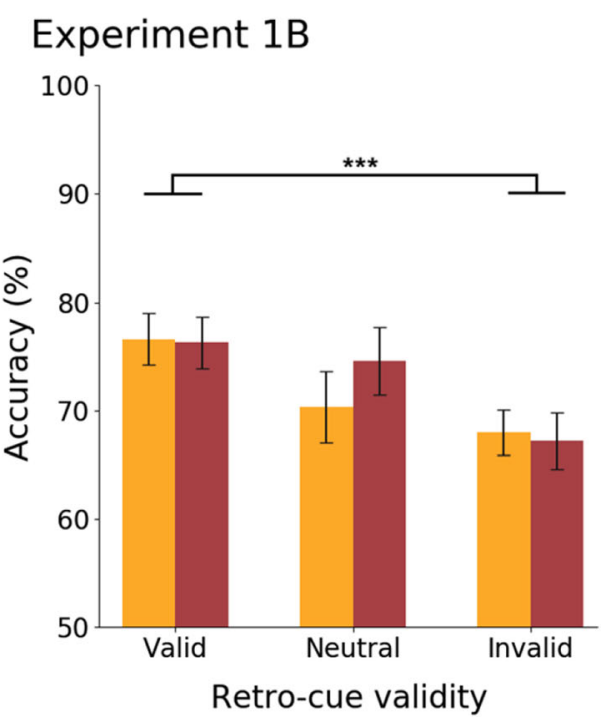

better in the valid cue condition than in the invalid cue condition. Error bars indicate one standard error of the mean. * $p<.05$; ** $p<.01$; *** $p<$ .001 
the above requirements. Therefore, we think that one had to retain the exact depth position of the target to correctly perform the task.

\section{Experiment 1b}

The results are shown in Fig. 2, right. The $2 \times 3$ repeatedmeasures ANOVA showed a significant main effect of retrocue validity, $F(2,42)=10.34, p<.001, \eta_{\mathrm{p}}{ }^{2}=.33$. Post hoc comparisons showed that the performance was significantly better in the valid retro-cue condition $(M=.76)$ than in the invalid retro-cue condition $(M=.68), t(21)=5.52, p<.001$. No significant difference was found between the valid cue and neutral cue conditions $(M=.72), t(21)=2.09, p=.147$, and between the neutral cue and invalid cue conditions, $t(21)=$ $2.14, p=.134$. The main effect of retro-cue type was not significant, $F(1,21)=.53, p=.475, \eta_{\mathrm{p}}{ }^{2}=.03$. No significant interaction was found, $F(2,42)=1.33, p=.275, \eta_{\mathrm{p}}{ }^{2}=.06$.

As in Experiment 1a, there was no significant invalid retrocue cost. However, the valid retro-cue benefit was also not significant. We found that the inconsistence can be mainly attributed to the surprisingly high accuracy of the 3D symbolic cue in the neutral cue condition. Additionally, the overall accuracies for 2D symbolic and 3D symbolic cues were not significantly different from each other. The nonsignificant main effect of cue type may indicate the memory benefit observed for 3D symbolic cues in Experiment 1a is weak and unstable, but it is unclear why accuracy of the 3D symbolic cues in the neutral cue condition was high and no significant benefit was observed in Experiment 1b. Since the stimuli and procedure were the same in the two experiments, the inconsistence might be due to sampling errors from an unlucky draw of sample. Therefore, further evidence on the effects of both retro-cue and cue type is needed to clarify these findings.

\section{Experiment 2}

In Experiment 2, we compared the effects of feature-based (color) cues and 3D symbolic cues to examine whether feature-based attention could produce similar retro-cue effects on WMd and to further clarify whether retro-cue benefits and costs can be reliably observed for WMd.

\section{Method}

\section{Participants}

Twenty-four students from SYSU with normal or correctedto-normal vision took part in the experiment. Written informed consent was obtained from each participant following a protocol approved by the IRB of SYSU.

\section{Stimuli and procedure}

The stimuli and procedure in Experiment 2 was identical to Experiment 1a, except for the following changes. Each square was of a different color, and the color of squares in the memory array was randomly chosen without replacement from six highly discriminable colors (red, green, blue, yellow, cyan, and magenta). Color retro-cues and 3D symbolic retro-cues (same as in Experiment 1a) were tested. The color retro-cue was a disk that subtended $0.15^{\circ} \times 0.15^{\circ}$ with a color identical to one of the memory items (see Fig. 3a). Observers completed a total of 480 trials with 240 trials for each retro-cue type. There were 24 invalid retro-cue trials, 192 valid retro-cue trials (retro-cue reliability 89\%), and 24 neutral cue trials. Different retro-cue types (color vs. 3D symbolic) were tested in separate experimental blocks, with randomized order across participants.

The methods for data analysis were the same as in Experiment 1a (see Supplemental Materials for reports on $d^{\prime}$ and BFs). Preliminary data screening showed that seven of the participants had accuracy below $50 \%$ in at least one of the experimental conditions; therefore, the data of these participants were excluded for analysis in this experiment.

\section{Results and discussion}

The results are shown in Fig. $3 \mathrm{~b}$. The $2 \times 3$ repeated-measure ANOVA showed a significant main effect of retro-cue validity, $F(2,32)=9.35, p=.001, \eta_{\mathrm{p}}{ }^{2}=.37$. Post hoc comparisons showed that the performance was significantly better for the valid cues $(M=.77)$ than for the neutral cues $(M=.70), t(16)=$ $3.18, p=.018$, and the invalid cues $(M=.69), t(16)=4.61, p$ $=.001$. No significant difference was found between the neutral and invalid cues, $t(16)=.29, p=1.00$. The main effect of retro-cue type was not significant, $F(1,16)=3.69, p=.073$, $\eta_{\mathrm{p}}{ }^{2}=.19$. No significant interaction was found, $F(2,32)=$ $1.41, p=.260, \eta_{\mathrm{p}}^{2}=.08$.

The results were generally consistent with Experiment $1 \mathrm{a}$. We found significant valid retro-cue benefits, but no significant cost. The overall memory performance was not significantly different between the 3D symbolic retro-cue and the feature-based retro-cue.

\section{Experiment 3}

The above experiments found significant retro-cue benefits, but no cost. Previous studies have shown that retro-cue reliability would influence the magnitude of valid retro-cue benefits and invalid retro-cue costs (Gunseli et al., 2015; Souza et al., 2015). The retro-cue benefits may decrease and the costs may disappear as cue reliability decreases. In Experiment 3, we carried out two experiments to explore whether and how 


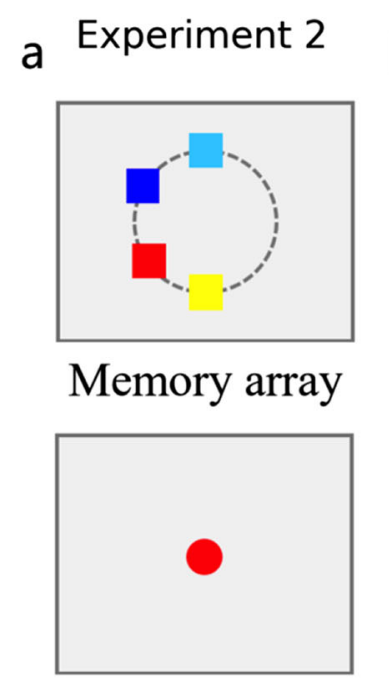

Color retro-cue

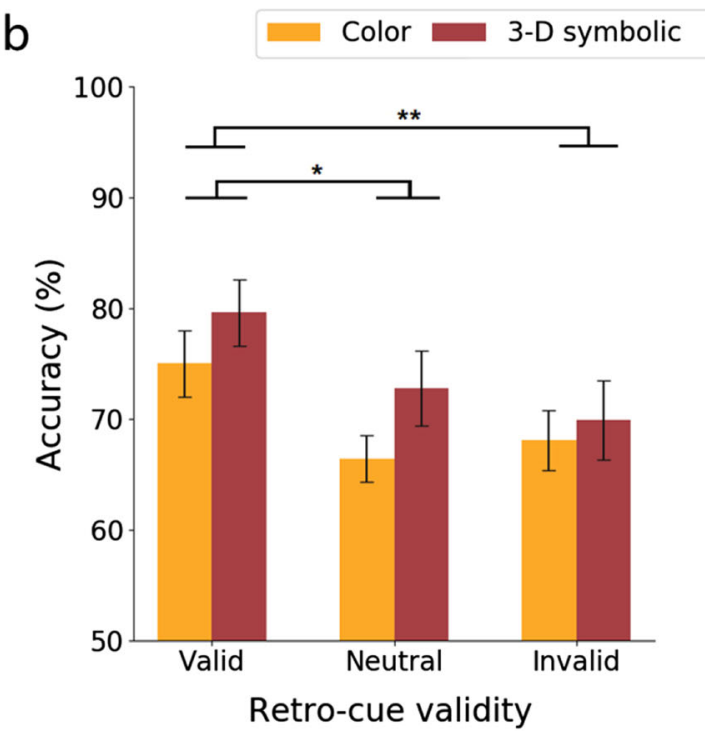

Fig. 3 Stimuli and results in Experiment 2. a Memory array and color retro-cue used in Experiment 2. b Mean accuracy for the color and 3D symbolic retro-cues. Significant retro-cue benefits were found, but retro-

manipulations on cue reliability could affect the retro-cue benefits and costs. We compared three types of retro-cues (color, arrow, and 3D symbolic), and the tested a cue reliability of $75 \%$ in Experiment $3 \mathrm{a}$ and of $50 \%$ in Experiment $3 \mathrm{~b}$. We expected to observe reduced retro-cue benefits as cue reliability decreases.

\section{Method}

\section{Participants}

Twenty-one students from SYSU with normal or corrected-tonormal vision took part in Experiment 3a and another 20 students participated in Experiment 3b. Written informed consent was obtained from each participant following a protocol approved by the IRB of SYSU.

\section{Stimuli and procedure}

The stimuli and procedure in Experiment 3 was identical to Experiment 2, except for the following changes. In both Experiments $3 \mathrm{a}$ and $3 \mathrm{~b}$, all three types of retro-cues (color, arrow, and 3D symbolic) were tested. In each experiment, observers completed a total of 480 trials with 240 trials for each retro-cue type. In Experiment 3a, there were 48 invalid retro-cue trials, 144 valid retro-cue trials (retro-cue reliability $75 \%, 144 / 192)$, and 48 neutral cue trials for each cue type; in Experiment 3b, there were 96 invalid retro-cue trials, 96 valid retro-cue trials (retro-cue reliability 50\%, 96/192), and 48 neutral cue trials for each cue type. There were 50\% 'change' trials in each experimental condition. Different retro-cue types

cue costs were not significant. Error bars indicate one standard error of the mean. $* p<.05 ; * * p<.01$

were tested in separate experimental blocks, with randomized order across participants.

Preliminary data screening showed that three participants in Experiment 3a and two participants in Experiment 3b had accuracy below $50 \%$ in at least one of the experimental conditions; therefore, the data of these participants were excluded for analysis. There were 18 participants included in each experiment for analysis. For each experiment, we performed a 3 $\times 3$ (retro-cue type $\times$ retro-cue validity) repeated-measures ANOVA on accuracy to examine the retro-cue effects (see Supplemental Materials for reports on $d^{\prime}$ and BFs). In addition, we further combined and compared the results of Experiments 2 and 3 to investigate how retro-cue benefits and costs changed with cue reliability. Since the memory array used in Experiment 1 (blue squares) was different from Experiments 2 and 3 (colored squares), we only used the data from Experiment 2 (89\% reliability, color and 3D symbolic cues) and Experiment 3 (75\% and 50\% reliability, color and $3 \mathrm{D}$ symbolic cues) to performed a $3 \times 2 \times 3$ (cue reliability: $89 \%$ vs. $75 \%$ vs. $50 \% \times$ retro-cue type: color vs. $3 \mathrm{D}$ symbolic $\times$ retro-cue validity: valid vs. neutral vs. invalid) mixed-design ANOVA. The $p$ values of post hoc tests were Bonferroni corrected.

\section{Results and discussion}

\section{Experiment 3a}

The results are shown in Fig. 4 , left. The $3 \times 3$ repeatedmeasures ANOVA showed a significant main effect of retro-cue validity, $F(2,34)=18.98, p<.001, \eta_{\mathrm{p}}{ }^{2}=.54$. Post hoc comparisons showed that the performance was 


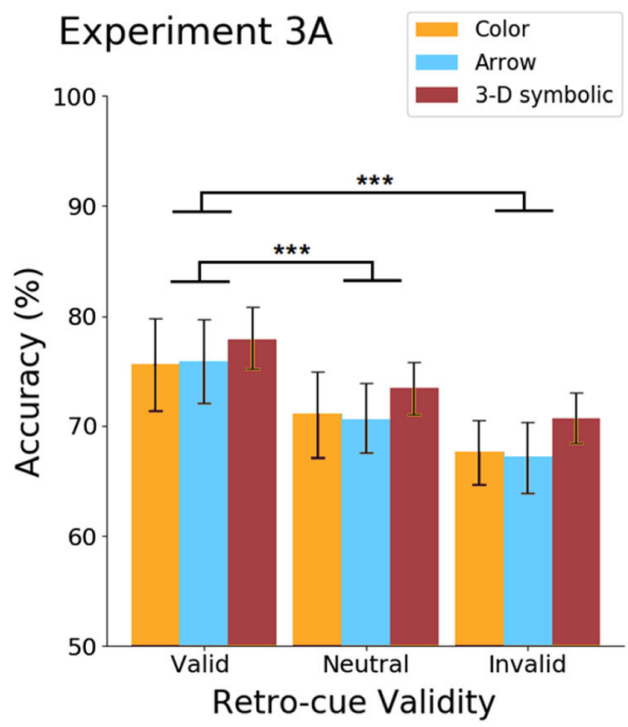

Fig. 4 Mean accuracy for the color, arrow and 3D symbolic retro-cues in Experiment $3 \mathrm{a}$ ( $75 \%$ valid) and $3 \mathrm{~b}$ (50\% valid). Significant retro-cue benefits were found in Experiment 3a, but not in Experiment 3b. No

significantly better for the valid cues $(M=.76)$ than for the invalid cues $(M=.69), t(17)=5.92, p<.001$, and the neutral cues $(M=.72), t(17)=3.84, p=.003$. No significant difference was found between the neutral and invalid cue conditions, $t(17)=2.25, p=.114$. There was no significant main effect of retro-cue type, $F(2,34)=1.56, p=$ $.225, \eta_{\mathrm{p}}{ }^{2}=.08$. No significant interaction was found, $F(4$, $68)=.15, p=.962, \eta_{\mathrm{p}}{ }^{2}<.01$. The results were consistent with Experiment 2.

\section{Experiment 3b}

The results are shown in Fig. 4, right. The results showed a significant main effect of retro-cue validity, $F(2,34)=5.83, p$ $=.007, \eta_{\mathrm{p}}{ }^{2}=.26$. Post hoc comparisons showed that the performance was significantly better for the valid cues $(M=.75)$ than for the invalid cues $(M=.72), t(17)=3.29, p$ $=.012$. No significant difference was found between the valid cues and neutral cues $(M=.74), t(17)=2.32, p=.099$, and between the neutral and invalid cues, $t(17)=.22, p=1.00$. The main effect of retro-cue type was not significant, $F(2,34)$ $=1.89, p=.166, \eta_{\mathrm{p}}^{2}=.10$. No significant interaction was found, $F(4,68)=1.91, p=.118, \eta_{\mathrm{p}}{ }^{2}=.10$. These results were consistent with Experiment 2, except that the valid retro-cue benefit was not significant.

The above experiments showed that the invalid retro-cue cost was reliably absent. The lack of significant difference in accuracy between the valid and neutral cues in Experiment $3 \mathrm{~b}$ may be attributed to the diminishing benefits as cue reliability decreased.

\section{Experiment 3B}

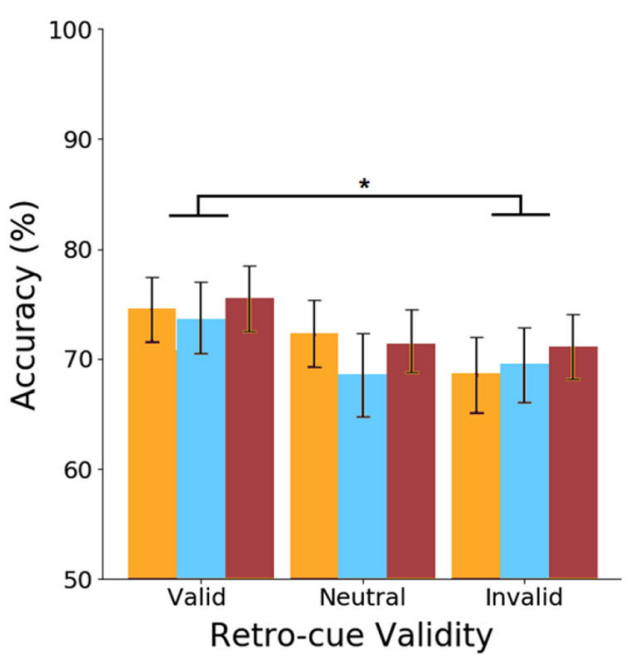

cost was found in both experiments. Error bars indicate one standard error of the mean. $* p<.05 ; * * p<.01 ; * * * p<.005$

\section{Comparison between cue reliability}

The results of $3 \times 2 \times 3$ mixed-design ANOVA showed a significant main effect of retro-cue validity, $F(2,100)=$ $27.38, p<.001, \eta_{\mathrm{p}}{ }^{2}=.35$. Post hoc comparisons showed that the performance was significantly better for the valid cues $(M$ $=.77)$ than for the neutral cues $(M=.72), t(52)=4.57, p<$ .001 , and the invalid cues $(M=.70), t(52)=7.46, p<.001$. No significant difference was found between the neutral and invalid cues, $t(52)=1.76, p=.273$. Surprisingly, there was a significant main effect of retro-cue type, $F(1,50)=4.42, p=$ $.041, \eta_{\mathrm{p}}{ }^{2}=.08$. Performance was significantly better for the 3D symbolic cues $(M=.74)$ than for the color cues $(M=.72)$. The main effect of retro-cue reliability was not significant, $F(2,50)=.14, p=.866, \eta_{\mathrm{p}}{ }^{2}<.01$. No significant interactions were found $(p s>.087)$.

By comparing the results across various cue reliability conditions, we found reliable valid retro-cue benefits, but no invalid retro-cue cost. The overall memory performance for different cue reliabilities was not significantly different from each other. Although the interaction between cue reliability and retro-cue validity was not significant ( $p=.087$ ), the observed valid retro-cue benefits increased with cue reliability. The mean benefit (valid - neutral) was about $2.40 \%$ in accuracy with a cue reliability of $50 \%$, about $4.84 \%$ with a cue reliability of $75 \%$, and about $7.49 \%$ with a cue reliability of $89 \%$. In addition, the overall performance for 3D symbolic retro-cue was significantly better than that for color retrocue. This effect was previously observed in Experiment 1a, but not in the others, so it is possible that the improvement in performance with 3D symbolic cues is quite weak, and only 
by pooling the data from three experiments could provide enough evidence to confirm its benefit.

\section{General discussion}

In the present study, we investigated whether there was an effect of attentional selection on WMd and whether different types of retro-cues differed in their effects on memory performance for depth. The results showed reliable and significant retro-cue benefits-memory performance for detecting changes in stereoscopic depths was improved for valid cue trials compared with those for neutral or invalid cue trials. In other words, selectively attending to a memory representation during retention could improve the memory performance for its depth position, which is similar to that reported for VWM (Berryhill et al., 2012; Gunseli et al., 2015; Shimi et al., 2014; Souza et al., 2015). Besides the 3D symbolic cues, cueing the color and the 2D spatial location of a memory item effectively enhanced performance for its depth representation, indicating that an object's depth representation was stored in association with its visual features and 2D location.

No significant invalid retro-cue cost was found in our study, even when the cue reliability was high ( $89 \%$ valid). The nonsignificant results cannot be due to the flooring effect, as the mean accuracy across the three experiments was about $72 \%$ for the neutral cue trials and about $70 \%$ for the invalidcue trials, which was well above the chance level. Although nonsignificant, one might notice that for all types of retrocues, the mean accuracy of the neutral trials was consistently and slightly higher than that of the invalid trials, showing a trace of costs. In order to further clarify the findings on costs, we performed separate repeated-measures ANOVAs for each type of cue, and the results consistently showed that no significant cost was observed $(p s>.064$, Bonferroni corrected; also see Supplemental Materials for BFs). It is possible that the potential cost was too weak to be observed for WMd with the current experimental settings. Previous studies on VWM have shown that the costs on invalid trials often can be observed with a highly reliable cue (i.e., $80 \%$ valid; Gunseli et al., 2015). Such costs can be attributed to the likely removal of the noncued items from VWM (Gunseli et al., 2015), or that the noncued items are maintained in memory but only in a less 'active' state (dual-state model; Dube, Lumsden, \& AlAidroos, 2018; Olivers, Peters, Houtkamp, \& Roelfsema, 2011). We suggest that the weak-to-none costs may be attributed to the fact that depth order is inherently stored in working memory. Previous research has shown that both metric and ordinal depths are represented in working memory (i.e., depth positions can be stored in reference to the others; Qian et al., 2020). In this study, even though the cued item receives more memory resources and its depth representations could be enhanced, retaining ordinal depth in memory protects the noncued items from complete removal or 'frozen' in a less active state. Since the precise metric depth representation may be lost but the ordinal representation, which provides coarse depth information, is preserved, the memory representation of the noncued items can be weakened yet not severe enough to produce significant costs.

While the lack of retro-cue costs in WMd may provide us insights on the way of how depth information is stored in working memory, the valid retro-cue benefits observed in our study may be attributed to strengthened depth representations by focusing attention on the cued item - the so-called 'refreshing' process (for review, see Souza \& Oberauer, 2016). Evidence on VWM suggests that the cued item may be increased in accessibility (Rerko \& Oberauer, 2013), possibly by strengthening the binding between the item and its context (e.g., its spatial location). The attentional strengthening (or the 'refreshing') theory is a prominent alternative account for explaining the retro-cue effects in VWM, in addition to the 'removal' theory. Furthermore, it has been shown that when attention is withdrawn from the cued item by instruction, valid retro-cue benefits are maintained, but invalid retrocue costs disappear (Li \& Saiki, 2014). However, in our study, we did not observe significant costs even with focused attention and high cue reliability, suggesting that retaining the relational information can 'silence' the removal process in $\mathrm{WMd}$, which in contrast indicates that the removal process can be a major account for the retro-cue costs observed in working memory.

The present study also compared the retro-cue effects of various types of cues. By pooling the data from Experiments 2 and 3, the results showed a weak benefit for using 3D symbolic cues, compared with feature-based color cues (see Comparison Between Cue Reliability section) and 2D spatial arrow cues (see results of Experiment 1a). We suggest two possible explanations for this benefit. One possibility is that cueing the relational depth information would allow participants to focus only on the dimension of depth, ignoring the other feature dimensions, such as color. Sticking to the task-relevant depth throughout the trial may reduce a processing cost incurred by switching between various stimulus dimensions (Found \& Müller, 1996), thereby improving the overall performance. Another possibility is that cueing the ordinal depth may facilitate the encoding and retention of metric depth representation, due to the storage mechanism of depth order. Even though ordinal depth representation is inherently stored regardless of retro-cue type, cueing depth order may further require the observers to encode the relative depth positions of items into explicit symbolic numbers on a semantic level to complete the task. Although the numbers per se did not carry precise metric depth information, the task might encourage a deeper level of processing during encoding; therefore, the depth 
positions were better stored. This explanation is consistent with a well-known phenomenon - the deeper the level of processing during initial perception, the better should be memory for the experience (Craik \& Tulving, 1975). Since the metric and ordinal depth representation may share the same memory store, cueing the ordinal depth during retention may also facilitate the consolidation process and retention of metric depth representation overall. Therefore, we observed a slight improvement on the memory performance for utilizing the information on depth order.

Previous research investigating the retro-cue effect on VWM sometimes examined the speed of access to memory representations, as measured by the reaction times (RTs) on correct trials, in addition to change detection accuracies (Souza \& Oberauer, 2016). A decrease in the RTs on the valid retro-cue trials compared with the neutral cue trials indicates the retro-cue benefits, and an increase in the RTs on the invalid retro-cue trials indicates the costs. Here, we did not analyze the RTs in this experiment for the following reasons. Because previous studies have shown that the memory performance for depth positions was poor (Qian \& Zhang, 2019), and the mean accuracies in our experiment were all below $80 \%$ for the valid retro-cue conditions and even lower for the other two conditions, the proportion of correct trials was low. Together with the trials that had excessively long RTs ( $>3$ s) and needed to be excluded (about 2.8\%), there were only about $68.7 \%, 62.9 \%$ and $59.4 \%$ of correct trials remaining for the valid, neutral, and invalid retro-cue conditions, respectively. Given such low proportions, we think RTs are not suitable to assess the retro-cue effects on WMd, and therefore they were not examined.

In summary, our study showed that memory performance for depth positions was improved for the cued item by using feature-based, spatial, and symbolic retro-cues, demonstrating reliable retro-cue benefits for WMd. The weak-to-none cost with high cue reliability provides supportive evidence that the memory representation for depth is inherently relational, and that depth order may be encoded and stored in WMd as well as metric depth. The attentional effect on memory representation in a $3 \mathrm{D}$ context is different from that in a $2 \mathrm{D}$ context, and the observed divergence in findings between VWM and WMd suggests the relational nature of depth information stored in working memory.

Supplementary Information The online version contains supplementary material available at https://oi.org/10.3758/s13421-020-01123-4.

Acknowledgement This work has been supported by the National Natural Science Foundation of China (31500919), and the Fundamental Research Funds for the Central Universities (20wkzd12). The authors have no competing financial interests that might be perceived to influence the results and/or discussion reported in this paper.
Open practices statement The data and materials for all experiments are available upon request and none of the experiments was preregistered.

\section{References}

Astle, D. E., Summerfield, J., Griffin, I., \& Nobre, A. C. (2012). Orienting attention to locations in mental representations. Attention, Perception, \&Psychophysics, 74, 146-162.

Baddeley, A. (2003). Working memory and language: An overview. Journal of Communication Disorders, 36(3), 189-208.

Berryhill, M. E., Richmond, L. L., Shay, C. S., \& Olson, I. R. (2012). Shifting attention among working memory representations: Testing cue type, awareness, and strategic control. The Quarterly Journal of Experimental Psychology, 65(3), 426-438.

Blakemore, C. (1970). The range and scope of binocular depth discrimination in man. The Journal of Physiology, 211(3), 599-622. https:// doi.org/10.1113/jphysiol.1970.sp009296

Chunharas, C., Rademaker, R. L., Sprague, T. C., \& Brady, T. F. (2019) Separating memoranda in depth increases visual working memory performance. Journal of Vision, 19(1), 4, 1-16.

Craik, F. I. M., \& Tulving, E. (1975). Depth of processing and the retention of words in episodic memory. Journal of Experimental Psychology: General, 104(3), 268-294.

Dube, B., Lumsden, A., \& Al-Aidroos, N. (2018). Probabilistic retro-cues do not determine state in visual working memory. Psychonomic Bulletin \& Review, 26(2), 641-646.

Faul, F., Erdfelder, E., Lang, A.-G., \& Buchner, A. (2007). G*Power 3: A flexible statistical power analysis program for the social, behavioral, and biomedical sciences. Behavior Research Methods, 39, 175-191.

Found, A., \& Müller, H. J. (1996). Searching for unknown feature targets on more than one dimension: Investigating a "dimensionweighting" account. Perception \& Psychophysics, 58(1), 88-101.

Griffin, I. C., \& Nobre, A. C. (2003). Orienting attention to locations in internal representations. Journal of Cognitive Neuroscience, 15(8), $1176-1194$.

Gunseli, E., Van Moorselaar, D., Meeter, M., \& Olivers, C. N. L. (2015). The reliability of retro-cues determines the fate of noncued visual working memory representations. Psychonomic Bulletin \& Review, 22(5), 1134-1341.

Heuer, A., \& Schubö, A. (2016). Feature-based and spatial attentional selection in visual working memory. Memory \& Cognition, 44(4), 621-632.

Heuer, A., Schubö, A., \& Crawford, J. D. (2016). Different cortical mechanisms for spatial vs. feature-based attentional selection in visual working memory. Frontiers in Human Neuroscience, 10(415), 1-9.

Kuo, B.-C., Stokes, M. G., \& Nobre, A. C. (2012). Attention modulates maintenance of representations in visual short-term memory. Journal of Cognitive Neuroscience, 24, 51-60. https://doi.org/10. 1162/jocn_a_00087

Lamy, D., \& Tsal, Y. (2001). On the status of location in visual attention. European Journal of Cognitive Psychology, 13(3), 305-342.

LaRocque, J. J., Lewis-Peacock, J. A., Drysdale, A. T., Oberauer, K., \& Postle, B. R. (2013). Decoding attended information in short-term memory: An EEG study. Journal of Cognitive Neuroscience, 25, $127-142$

Lewis-Peacock, J. A., Drysdale, A. T., Oberauer, K., \& Postle, B. R. (2011). Neural evidence for a distinction between short-term memory and the focus of attention. Journal of Cognitive Neuroscience, 24(1), 61-79.

Li, Q., \& Saiki, J. (2014). The effects of sequential attention shifts within visual working memory. Frontiers in Psychology, 5, 965.

Luck, S. J., \& Vogel, E. K. (1997). The capacity of visual working memory for features and conjunctions. Nature, 390(6657), 279281. 
Luck, S. J., \& Vogel, E. K. (2013). Visual working memory capacity: from psychophysics and neurobiology to individual differences. Trends in Cognitive Sciences, 17(8), 391-400.

McMains, S. A., Fehd, H. M., Emmanouil, T. A., \& Kastner, S. (2007). Mechanisms of feature-and space-based attention: Response modulation and baseline increases. Journal of Neurophysiology, 98(4), 2110-2121.

Murray, A. M., Nobre, A. C., Clark, I. A., Cravo, A. M., \& Stokes, M. G. (2013). Attention restores discrete items to visual short-term memory. Psychological Science, 24(4), 550-556.

Olivers, C. N., Peters, J., Houtkamp, R., \& Roelfsema, P. R. (2011). Different states in visual working memory: When it guides attention and when it does not. Trends in Cognitive Sciences, 15(7), 327-334.

Poch, C., Carretie, L., \& Campo, P. (2017). A dual mechanism underlying alpha lateralization in attentional orienting to mental representation. Biological Psychology, 128, 63-70.

Qian, J., Li, J., Wang, K., Liu, S., \& Lei, Q. (2017). Evidence for the effect of depth on visual working memory. Scientific Reports, 7(1), 6408.

Qian, J., Li, Z., Zhang, K., \& Lei, Q. (2020). Relation matters: Relative depth order is stored in working memory for depth. Psychonomic Bulletin \& Review, 1-9. Advance online publication.

Qian, J., \& Zhang, K. (2019). Working memory for stereoscopic depth is limited and imprecise-Evidence from a change detection task. Psychonomic Bulletin \& Review, 26, 1657-1665.

Qian, J., Zhang, K., Lei, Q., Han, Y., \& Li, W. (2019). Task-dependent effects of voluntary space-based and involuntary feature-based attention on visual working memory. Psychological Research, 84, 1304-1319.

Qian, J., Zhang, K., Wang, K., Li, J., \& Lei, Q. (2018). Saturation and brightness modulate the effect of depth on visual working memory. Journal of Vision, 18(9), 16, 1-12.

Reeves, A., \& Lei, Q. (2014). Is Visual Short-term memory depthful? Vision Research, 96, 102-112.

Reeves, A., \& Lei, Q. (2017). Short-term visual memory for location in depth: A U-shaped function of time. Attention, Perception, \& Psychophysics, 79, 1917-1932

Rerko, L., \& Oberauer, K. (2013). Focused, unfocused, and defocused information in working memory. Journal of Experimental Psychology: Learning, Memory, and Cognition, 39, 1075-1096.
Shimi, A., Nobre, A. C., Astle, D. E., \& Scerif, G. (2014). Orienting attention within visual short-term memory: Development and mechanisms. Child Development, 85, 578-592.

Simons, D. J. (1996) In sight, out of mind: When object representations fail. Psychological Science, 7(5), 301-305.

Souza, A. S., \& Oberauer, K. (2016). In search of the focus of attention in working memory: 13 years of the retro-cue effect. Attention, Perception, \& Psychophysics, 78(7), 1839-1860.

Souza, A. S., Rerko, L., \& Oberauer, K. (2015). Refreshing memory traces: Thinking of an item improves retrieval from visual working memory. Annals of the New York Academy of Sciences, 1339, 20 31 .

Theeuwes, J. (1989). Effects of location and form cuing on the allocation of attention in the visual field. Acta Psychologica, 72(2), 177-192.

van Moorselaar, D., Olivers, C. N. L., Theeuwes, J., Lamme, V., \& Sligte, I. G. (2015). Forgotten but not gone: Retro-cue costs and benefits in a double-cueing paradigm suggest multiple states in visual shortterm memory. Journal of Experimental Psychology: Learning, Memory, and Cognition, 41(6), 1727-1727.

Wang, B., Theeuwes, J., \& Olivers, C. N. L. (2018). When shorter delays lead to worse memories: Task disruption makes visual working memory temporarily vulnerable to test interference. Journal of Experimental Psychology: Learning, Memory, and Cognition, 44(5), 722-733.

Wang, B., Yan, C., Wang, Z., Olivers, C. N. L., \& Theeuwes, J. (2017). Adverse orienting effects on visual working memory encoding and maintenance. Psychonomic Bulletin \& Review, 24(4), 1261-1267. https://doi.org/10.3758/s13423-016-1205-4

Xu, Y., \& Nakayama, K. (2007). Visual short-term memory benefit for objects on different 3D surfaces. Journal of Experimental Psychology: General, 136(4), 653-662.

Zhang, K., Gao, DG. \& Qian, J. (2020). Overestimation and contraction biases of depth information stored in working memory depend on spatial configuration. British Journal of Psychology, 1-13.

Publisher's note Springer Nature remains neutral with regard to jurisdictional claims in published maps and institutional affiliations. 